\title{
Heart Failure Risk Reduction: Hydrophilic or Lipophilic Statins?
}

\author{
Setor K. Kunutsor ${ }^{\mathrm{a}} \mathrm{b}$ Jari A. Laukkanen ${ }^{\mathrm{c}-\mathrm{e}}$ \\ ${ }^{a}$ National Institute for Health Research Bristol Biomedical Research Centre, University Hospitals Bristol NHS \\ Foundation Trust and University of Bristol, Bristol, UK; ${ }^{\mathrm{b}}$ Musculoskeletal Research Unit, Translational Health \\ Sciences, Learning and Research Building, Southmead Hospital, Bristol Medical School, University of Bristol, Bristol, \\ UK; ' ${ }^{C}$ Faculty of Sport and Health Sciences, University of Jyväskylä, Jyväskylä, Finland; ' Institute of Public Health and \\ Clinical Nutrition, University of Eastern Finland, Kuopio, Finland; ${ }^{~}$ Department of Medicine, Central Finland \\ Health Care District, Jyväskylä, Finland
}

Heart failure (HF), which is the end stage of most cardiovascular diseases (CVDs) [1], is a global public health burden, which is associated with substantial morbidity, mortality as well as health care costs [2]. In the USA alone, HF contributes to about USD 31 billion in annual healthcare expenditure [3]. With recent advances in the treatment of CVDs that have led to their decline, there has been an increase in the prevalence of HF over the last few decades [4]. The increasing prevalence of HF has been reported to reflect a combination of factors such as aging of the population and improvements in the treatment of CVDs [4]. In the USA, HF affects approximately 6.5 million Americans [5], and it has been projected that 8 million people will be affected by 2030.[5] HF has considerable impact on both the economy and society; hence HF prevention strategies should be urgently considered.

According to a report from the American Heart Association (AHA), optimal profiles in smoking, body mass index (BMI), physical activity, diet, blood pressure, glucose as well as cholesterol are associated with a lower lifetime risk of HF [5]. The 3-hydroxy-3-methylglutaryl coenzyme A reductase inhibitors (statins), are established for the primary and secondary prevention of CVD, and this is based on their lipid-lowering properties [6-8]. Statins promote lipid-lowering by inhibiting cholesterol biosynthesis and enhancing low-density lipoprotein clearance from the circulation. Beyond their lipid-lowering properties, statins are also suggested to have several pleiotropic effects, and these include decreasing oxidative stress and inflammation, improving endothelial function, enhancing stability of atherosclerotic plaques, decreasing platelet activation, inhibiting thrombosis, and inhibition of smooth muscle proliferation $[9,10]$. Statins have been reported to lower the HF risk by reducing the risk and progression of coronary heart disease (via their cholesterol-lowering effects) [11, 12]; they also have a beneficial effect on clinical outcomes in HF patients [13], which may be via some of their pleiotropic effects. Statins are classified into hydrophilic and lipophilic groups based on tissue selectivity. Lipophilic statins are widely distributed in different tissues, whereas hydrophilic statins are liver specific [14]. Lipophilic statins include atorvastatin, simvastatin, lovastatin, fluvastatin, cerivastatin and pitavastatin, while hydrophilic statins include rosuvastatin and pravastatin. Hydrophilic statins employ carrier-mediated mechanisms for uptake, which could reduce their ability to exert non-lipid effects on extrahepatic tissues [14], and they are suggested to be less pleiotropic. Though a headto-head comparison is not yet available, pooled data based on an indirect comparison approach suggests that lipophilic statins have more beneficial effects on HF-re- karger@karger.com

www.karger.com/crd

Karger
(C) 2020 S. Karger AG, Basel

(n)
Setor K. Kunutsor

Translational Health Sciences, Learning and Research Building (Level 1)

Southmead Hospital, Bristol Medical School, University of Bristol

Bristol BS10 5NB (UK)

skk31@ cantab.net 
lated clinical outcomes compared to hydrophilic statins (rosuvastatin) [15].

Based on the limited data regarding the clinical impact of different statins on future HF risk according to lipophilicity, Imran et al. [16] present novel data comparing the risk of incident HF between hydrophilic and lipophilic statins. Using a large observational cohort based on claims data of approximately 8 million patients with a mean age of 58 years, the risk of incident HF between initiators of hydrophilic statins and those of lipophilic statins was compared after propensity matching. Effect estimates for HF were also estimated separately for low- and high-intensity users. After a median follow-up of 2 years, 8,389 incident HF cases were recorded. The results suggested a modest reduction in HF for hydrophilic statins compared with lipophilic statins, which seemed to be driven by high-dose rather than low-dose hydrophilic statins. There was no evidence of effect modification on the association by sex, gender, and specific statins (rosuvastatin versus atorvastatin). Furthermore, the results appeared to be robust in a number of sensitivity analyses. The authors postulated several reasons for the difference in HF risk between hydrophilic and lipophilic statins, and these included differing pleiotropic effects and solubilities, cytokine action, and glucose homeostasis. Given that these reasons were mainly speculative, the authors acknowledged the association between statin lipophilicity and HF risk to be a complex one and called for further investigation to elucidate the potential pathways [16].

The strengths of the current study include the novelty, the large sample size with use of real world data and its generalizability to the United States population, use of a comprehensive panel of covariates with utilization of propensity score matching, incorporation of a lag of 1 year to reduce the potential misclassification of previously subclinical HF, and several sensitivity analyses to ensure robustness of results. Despite the adjustment for several covariates and using propensity matching, there was still a potential for residual confounding given the observational design. Another limitation was the inability to adjudicate HF events. In light of the limited data available, the authors called for more research in other populations.

Despite the modest risk reduction associated with use of hydrophilic compared with lipophilic statins, the current findings are very timely and relevant [16]. Though statins have been reported to reduce the future risk of HF, it appears this effect may be driven by high-dose hydrophilic statins such as rosuvastatin. The clinical question is should statins be prescribed to reduce the risk of HF based on lipophilicity? It may be too early to say so as the current evidence is based on a single observational cohort. Whether hydrophilic statins would be beneficial for HF prevention needs to be resolved in future studies. More importantly, definitive interventional evidence is needed to corroborate these early findings. Nevertheless, we commend the authors for putting together this fine study on the comparative effects of hydrophilic and lipophilic statins on future HF risk. Indeed, as the authors have clearly acknowledged in their conclusions, further work is needed. This investigation should stimulate further studies in the topic area. The potential relevance of statin lipophilicity in HF prevention may have some clinical implications, but the AHA reports that the primary prevention of HF can be augmented by greater adherence to its "My Life Check - Life's Simple 7 goals", which involve improving 7 major risk factors (smoking, BMI, physical activity, diet, blood pressure, glucose, and cholesterol) through lifestyle changes, and this remains the cornerstone of HF prevention.

\section{Disclosure Statement}

The authors declare that they do not have any conflict of interest.

\section{Funding Sources}

Dr. Kunutsor acknowledges support from the NIHR Biomedical Research Centre at University Hospitals Bristol NHS Foundation Trust and the University of Bristol. The views expressed in this publication are those of the authors and not necessarily those of the NHS, the National Institute for Health Research or the Department of Health and Social Care. These sources had no role in the design and conduct of the study; collection, management, analysis, and interpretation of the data; and preparation, review, or approval of the manuscript.

References

1 Huang H, Huang B, Li Y, Huang Y, Li J, Yao $\mathrm{H}$, et al. Uric acid and risk of heart failure: a systematic review and meta-analysis. Eur J Heart Fail. 2014 Jan;16(1):15-24.

2 Yancy CW, Jessup M, Bozkurt B, Butler J, Casey DE Jr, Drazner MH, et al.; American College of Cardiology Foundation; American Heart Association Task Force on Practice Guidelines. 2013 ACCF/AHA guideline for the management of heart failure: a report of the American College of Cardiology Foundation/American Heart Association Task Force on Practice Guidelines. J Am Coll Cardiol. 2013 Oct;62(16):e147-239. 
3 Khera R, Pandey A, Ayers CR, Agusala V, Pruitt SL, Halm EA, et al. Contemporary Epidemiology of Heart Failure in Fee-For-Service Medicare Beneficiaries Across Healthcare Settings. Circ Heart Fail. 2017 Nov; 10(11):e004402.

4 Heidenreich PA, Albert NM, Allen LA, Bluemke DA, Butler J, Fonarow GC, et al.; American Heart Association Advocacy Coordinating Committee; Council on Arteriosclerosis, Thrombosis and Vascular Biology; Council on Cardiovascular Radiology and Intervention; Council on Clinical Cardiology; Council on Epidemiology and Prevention; Stroke Council. Forecasting the impact of heart failure in the United States: a policy statement from the American Heart Association. Circ Heart Fail. 2013 May;6(3):606-19.

5 Benjamin EJ, Virani SS, Callaway CW, Chamberlain AM, Chang AR, Cheng S, et al.; American Heart Association Council on Epidemiology and Prevention Statistics Committee and Stroke Statistics Subcommittee. Heart Disease and Stroke Statistics-2018 Update: A Report From the American Heart Association. Circulation. 2018 Mar;137(12):e67-492.

6 Heart Protection Study Collaborative Group. MRC/BHF Heart Protection Study of cholesterol lowering with simvastatin in 20,536 high-risk individuals: a randomised placebocontrolled trial. Lancet. 2002 Jul;360(9326): $7-22$.
7 Ridker PM, Danielson E, Fonseca FA, Genest J, Gotto AM Jr, Kastelein JJ, et al.; JUPITER Study Group. Rosuvastatin to prevent vascular events in men and women with elevated C-reactive protein. N Engl J Med. 2008 Nov; 359(21):2195-207.

8 Stone NJ, Robinson JG, Lichtenstein AH, Bairey Merz CN, Blum CB, Eckel RH, et al.; American College of Cardiology/American Heart Association Task Force on Practice Guidelines. 2013 ACC/AHA guideline on the treatment of blood cholesterol to reduce atherosclerotic cardiovascular risk in adults: a report of the American College of Cardiology/ American Heart Association Task Force on Practice Guidelines. J Am Coll Cardiol. 2014 Jul;63(25 25 Pt B):2889-934.

9 Liao JK. Effects of statins on 3-hydroxy3-methylglutaryl coenzyme a reductase inhibition beyond low-density lipoprotein cholesterol. Am J Cardiol. 2005 Sep;96(5 5A): $24 \mathrm{~F}-33 \mathrm{~F}$.

10 Kunutsor SK, Whitehouse MR, Blom AW, Laukkanen JA. Statins and venous thromboembolism: do they represent a viable therapeutic agent? Expert Rev Cardiovasc Ther. 2017 Aug;15(8):629-37.
11 Kjekshus J, Pedersen TR, Olsson AG, Faergeman $\mathrm{O}$, Pyörälä K. The effects of simvastatin on the incidence of heart failure in patients with coronary heart disease. J Card Fail. 1997 Dec;3(4):249-54.

12 Long-Term Intervention with Pravastatin in Ischaemic Disease (LIPID) Study Group. Prevention of cardiovascular events and death with pravastatin in patients with coronary heart disease and a broad range of initial cholesterol levels. N Engl J Med. 1998 Nov; 339(19):1349-57.

13 Bielecka-Dabrowa A, Bytyçi I, Von Haehling S, Anker S, Jozwiak J, Rysz J, et al. Association of statin use and clinical outcomes in heart failure patients: a systematic review and metaanalysis. Lipids Health Dis. 2019 Oct;18(1): 188.

14 Schachter M. Chemical, pharmacokinetic and pharmacodynamic properties of statins: an update. Fundam Clin Pharmacol. 2005 Feb; 19(1):117-25.

15 Bonsu KO, Reidpath DD, Kadirvelu A. Lipophilic Statin Versus Rosuvastatin (Hydrophilic) Treatment for Heart Failure: a MetaAnalysis and Adjusted Indirect Comparison of Randomised Trials. Cardiovasc Drugs Ther. 2016 Apr;30(2):177-88.

16 Imran T, Wong A, Schneeweiss S, Desai RJ. Statin lipophilicity and the risk of incident heart failure. Cardiology. DOI: 10.1159/ 000506003 